\title{
A Century after the Braggs - On Locating and Refining H-Atoms Using X- Rays and Neutrons
}

\author{
Krzysztof Woźniak ${ }^{a}$ \\ aDepartment of Chemistry, University of Warsaw, Pasteura 1, 02-093 Warszawa, Poland, \\ kwozniak@chem.uw.edu.pl
}

\begin{abstract}
Hydrogen - a chemical element with an atomic number of 1 - is absolutely essential to life. Hydrogen makes up two of the three atoms in water and is present in all organic compounds. Covalently bonded $\mathrm{H}$ atoms involved in an interaction with another adjacent atom bearing a lone pair of electrons forms hydrogen bonding. Hydrogen bonding plays a key role in determining the three-dimensional structures and properties of DNA and proteins. The hydrogen nucleus, a proton, carries a unit positive electric charge and plays a particularly important role in acid-base reactions while in ionic compounds, hydrogen can take the form of a negative ion known as a hydride. Studies of the energetics and bonding of the hydrogen atom played a key role in the development of quantum mechanics. Accurately locating and refining $\mathrm{H}$ atoms is crucial in many fields of modern science. Because they have only one electron, hydrogen atoms are very hard to detect accurately with X-rays because X-rays are scattered by electron density. However, thermal neutrons diffract strongly from the nuclei of $\mathrm{H}$ atoms. This makes $\mathrm{H}$-atoms readily detectable by neutron radiation from nuclear reactors or spallation facilities.

There is quite a paradox that a century after the introduction of the Independent Atom Model (IAM - Bragg, 1914), 97\% of all ca. 1.5mln known crystal structures have been refined using the IAM which has some methodological deficiencies. In the IAM atoms are spheres which do not exchange electron density. In my presentation, I will present different approaches to localization and refinement of $\mathrm{H}$-atoms including applications of spherical Independent Atom Model [1] and aspherical approaches such as the Hansen-Coppens pseudoatom formalism applied in multipole modeling [2] and Hirshfeld Atom Refinement (HAR) [3]. I will present a detailed comparison of the results obtained with different approaches as a function of data resolution and models of electron density and compare these with the results of neutron diffraction and theoretical computations. I will discuss the accuracy and precision of different hydrogen atom bonds. Additionally, I will present how $\mathrm{H}$-atom ADPs change with data resolution and how the electron density was modeled. A century after the Braggs, it is now possible to obtain $\mathrm{H}$-atom positions from $\mathrm{X}$-ray studies which are equally reliable as those from neutron diffraction.
\end{abstract}

\section{Reference}

[1] Sanjuan-Szklarz, W. F., Hoser, A. A., Gutmann, M., Madsen A. Ø. \& Woźniak K. (2016) IUCr Journal, 3, 61-70.

[2] Hoser, A. A., Dominiak P. M. \& Woźniak K. (2009) Acta Crystallographica, A65 (2009) 300-311.

[3] Woińska, M., Grabowsky, S., Dominiak, P. M., Woźniak, K. \& Jayatilaka D. (2016).

Science Advances, 2 No. 5, e1600192. 